\title{
Identification of the Quantitative Trait Loci in Japonica Rice Landrace Heikezijing Responsible for Broad-Spectrum Resistance to Rice Blast
}

\author{
Xiaoliang Shi, Jianfei Wang, Yongmei Bao, Peifu Li, Liujie Xie, Ji Huang, and Hongsheng Zhang
}

National Key Laboratory of Crop Genetics \& Germplasm Enhancement, College of Agriculture, Nanjing Agricultural University, Nanjing 210095, China.

Accepted for publication 6 April 2010.

\section{ABSTRACT}

Shi, X., Wang, J., Bao, Y., Li, P., Xie, L., Huang, J., and Zhang, H. 2010. Identification of the quantitative trait loci in japonica rice landrace Heikezijing responsible for broad-spectrum resistance to rice blast. Phytopathology 100:822-829.

Rice blast is one of the most devastating diseases affecting rice production worldwide. One japonica landrace, Heikezijing, from the Taihu Lake area in China, has been reported to be highly resistant to most of the rice blast isolates collected from China and Japan. To effectively dissect the inheritance of its resistance, a population of recombinant inbred lines (RILs) $\left(\mathrm{F}_{2: 8}\right)$ was constructed from a cross between Heikezijing and Suyunuo, a blast-susceptible cultivar. Nineteen blast isolates from China and Japan were inoculated into 166 RILs and their parents, and 22 quantitative trait loci (QTLs) conferring resistance to these isolates were identified and mapped onto rice chromosomes 1, 7, 9, 11, and 12. Most of the QTLs conferred race-specific resistance to blast. Some QTLs, such as qtl11-5-5, conferred resistance to two or more isolates. One blast-resistant gene cluster, including qtl11-2-2, qtl11-3-1, qtl11-4-1, qtl11-5-5, qtl11-6-1, qtl11-7-5, qtl11-8-2, qtl11-9-2, qtl11-104 , and qtl11-11-1, was found on the long arm of chromosome 11 in the japonica landrace. These loci offered effective resistance toward as many as 17 isolates, including 16 isolates from seven Chinese race groups and 1 isolate from Japan. The results from this study suggest that the Heikezijing landrace involves a number of genes that are associated with broad-spectrum resistance to rice blast.
Rice blast, caused by the fungus Magnaporthe grisea (T. T. Hebert) M. E. Barr (anamorph, Pyricularia grisea (Cooke) Sacc), is one of the most devastating diseases affecting rice, the staple food for the largest portion of the world population (48). Many resistant rice cultivars often remain effective only a few years because of the genetic instability and pathogenic variability of $M$. grisea (31). However, the breeding of resistant rice remains the most promising choice in order to avoid an epidemic outbreak of rice blast $(5,47)$. It has been a major goal to find and characterize effective resistance genes from rice, and to map and clone these genes for breeding resistant plants.

To date, $>50$ blast-resistant genes with qualitative traits have been reported. These genes are distributed on 11 of the 12 rice linkage groups, with the exception of chromosome 3 , and many of them are clustered on chromosomes 6,11 , and $12(54,64)$. Most of the resistance genes are dominant, except the recessive gene pi21. Thus far, 13 genes resistant to rice blast (Pib, Pi-ta, Pi9, Pi2, Piz-t, Pi-d2, Pi36, Pi37, Pikh, Pit, Pik-m, Pi5, and pi21) have been isolated through a map-based cloning approach $(2,6,10$, $14,15,21,24,28,41,44,53)$. Among these cloned genes, 11 genes belong to a large resistance $(R)$ gene family encoding receptor proteins that contain nucleotide-binding sites (NBSs) and leucinerich repeats (LRRs). In particular, the blast resistance mediated by

Corresponding authors: J. Huang and H. Zhang;

E-mail addresses: huangji@njau.edu.cn and hszhang@njau.edu.cn

X. Shi, J. Wang, and Y. Bao contributed equally to this work.

* The $\boldsymbol{e}$-Xtra logo stands for "electronic extra" and indicates that the online version contains three additional tables and one figure not available in the print version.

doi:10.1094/PHYTO-100-8-0822

(C) 2010 The American Phytopathological Society
Pik-m and Pi5 require the cooperation of two NBS-LRR genes $(2,21)$. $P i-d 2$, however, encodes a receptor-like kinase protein with a predicted extracellular domain of a bulb-type mannosespecific binding lectin (B-lectin) and an intracellular serine-threonine kinase domain (10). Interestingly, a single amino acid difference in the Pita protein correlates with the gene-for-genespecific characteristic of the Pita/AVR-Pita system (6). It has also been found that only a single amino acid change in $P i-d 2$ is responsible for the difference between resistant and susceptible alleles (10). The only recessive gene conferring non-race-specific resistance, pi21, encodes a proline-rich protein with a putative heavy-metal-binding domain and putative protein-protein interaction motifs. The loss of function of pi21 is likely due to the absence of the 18- and 48-bp PxxPxxP sequences (14).

Broad-spectrum disease $R$ genes have been used for disease control in crops. Broad-spectrum disease resistance can be achieved in two ways: resistance to the majority of geographically different isolates of the same pathogen or resistance to two or more unrelated pathogens (41). To date, several broad-spectrum $R$ genes have been cloned in plants $(7,14,41,50,55)$. In rice, some broad-spectrum rice blast resistance genes belonging to the first category, such as Pi1, Pi2, Pi9, Piz-t, Pi33, and Pigm(t), have been identified. These are all resistant to a number of rice blast isolates $(4,11,35,41,61)$. Genes such as Pi34 (58), Pi35(t) (38), and pi21 (14) confer partial resistance to blast. Recently, Manosalva et al. (32) reported a broad-spectrum disease resistance gene from indica cv. Sanhuangzhan 2 that is effective against two distinct, important rice pathogens.

In a previous study, we found that a japonica rice landrace, Heikezijing from the Taihu Lake region in China, exhibited a high level of resistance to nine blast isolates (51). Here, we identified resistance quantitative trait loci (QTLs) to 19 isolates in Heikezijing with a simple-sequence repeat (SSR) molecular linkage map. 


\section{MATERIALS AND METHODS}

Plant materials. Two rice (Oryza sativa L.) accessions were used in this study. The japonica landrace, Heikezijing, is highly resistant to the blast, and Suyunuo is susceptibility to most blast isolates $(22,51)$ The population of recombinant inbred lines $(\mathrm{RILs})\left(\mathrm{F}_{2: 8}\right)$ from the cross of Heikezijing $\times$ Suyunuo consisted of 166 lines.

Pathogens. Eighteen Chinese blast isolates from all seven races (ZA, ZB, ZC, ZD, ZE, ZF, and ZG), based on the Chinese Rice Blast Identification System (25), provided by the Plant Protection Research Institute, Jiangsu Academy of Agricultural Sciences, and one Japanese isolate, Hoku 1, provided by the Institute of Crop Research, Chinese Academy of Agricultural Sciences, were used in this study (Table 1).

Inoculation. Rice seeds were grown in plastic trays of 60 by 30 by $5 \mathrm{~cm}$ as described by Wang et al. (51). In each tray, 30 lines and 2 parents $\left(\mathrm{P}_{1}\right.$ and $\left.\mathrm{P}_{2}\right)$ as control were sown, with 10 seeds per line or parents for each inoculation. Three-week-old rice seedlings were put into inoculation chambers and inoculated by spraying with conidial suspensions $\left(5 \times 10^{4}\right.$ conidia/ml $)$ with several drops of Tween-20 as described by Wang et al. (51). The inoculated plants were kept in chambers at $26^{\circ} \mathrm{C}$ with $95 \%$ relative humidity and darkness for $24 \mathrm{~h}$, and then transferred to the greenhouse for incubation with $100 \%$ relative humidity by intermittently spraying with water. Each line was inoculated in two independent experiments, with two replications of each experiment. The lesion types on rice leaves were scored after 1 week of inoculation, according to standard procedures (31). Lesion scores of 0 to 5 were based on lesion types with appropriate reference of the disease area of the highest score of each plant, and the average lesion score of 10 plants was recorded as the final score of each line. RILs with scores of 0 to 2 were considered to be resistant and those with scores of 3 to 5 were considered to be susceptible. For QTL mapping, the average score of each RIL was used as a quantitative variable (43).

Construction of molecular linkage map. To construct the molecular linkage map, 0.2 to $0.5 \mathrm{~g}$ of leaves from RILs $\left(\mathrm{F}_{2: 7}\right)$ and parents were collected specifically for DNA extraction by the sodium dodecyl sulfate (SDS) method (40). According to the International Rice Microsatellite Initiative (IRMI; http://www. gramene.org), 1,436 SSR markers were used in this study, and 128 of them with polymorphisms between the two parents were used for map construction. Polymerase chain reaction (PCR) amplifications were carried out in $10-\mu \mathrm{l}$ reaction mixtures containing $0.25 \mu \mathrm{m}$ primer, Taq polymerase (Tiangen, Beijing) at $0.01 \mathrm{U} / \mu \mathrm{l}, 1 \times$ buffer mix, and $250 \mu \mathrm{m}$ dNTP. The PCR conditions was as follows: predenaturing at $94^{\circ} \mathrm{C}$ for $5 \mathrm{~min}$; followed by 35 cycles of denaturing at $94^{\circ} \mathrm{C}$ for $40 \mathrm{~s}$, annealing at $55^{\circ} \mathrm{C}$ for $40 \mathrm{~s}$, and extension at $72^{\circ} \mathrm{C}$ for $45 \mathrm{~s}$; and a final extension at $72^{\circ} \mathrm{C}$ for 10 min. The PCR products were separated on $8 \%$ nondenaturant polyacrylamide gels followed by a silver staining method according to the manufacturer's recommendations (Promega Corp., Madison, WI). A genetic map of molecular markers was computed using the multipoint functions performed by MapMaker/ EXP v. 3.0 (20). The logarithm of the odds (LOD) score threshold was set to 3.0. Ordering of markers was achieved using the "order", "try", and "ripple" commands, which calculate likelihood ratios for the different possible multipoint orders. Conversion of recombination fractions into centimorgans (cM) was obtained with Kosambi's mapping function (18). The final map was drawn using MapDraw 2.1 (27).

Data analysis. Composite interval mapping (CIM) (59) was performed for QTL analysis and CIM was computed using Windows QTL Cartographer 2.5 (52). A stringent LOD threshold of 3.0 was used to declare major QTLs in this study while a LOD score value of 2.5 was used to detect minor QTLs, as suggested by Lander (19). Mapmaker/QTL (20) and QTLnetwork 2.0 (56) were also used to confirm putative loci affecting blast resistance based on point and interval analysis and mixed-model-based CIM (MCIM). In this study, QTLs were named $q t l A-B-C$, in which A means the chromosome number, B the QTL, and $\mathrm{C}$ the number of isolates corresponding to the QTL.

\section{RESULTS}

Resistance spectrum of Heikezijing to blast. Nineteen blast isolates from China and Japan were inoculated onto Heikezijing, Suyunuo, and 166 RILs derived from the cross Heikezijing $\times$ Suyunuo. The results show that Heikezijing is resistant to all isolates, with lesion scores of 0 to 1.3 , and that Suyunuo is susceptible to all isolates, with lesion scores of 4 to 5 (Table 1). The RIL population showed different effects from the blast isolates, and the distribution frequency of lesion scores differed depending on the blast isolate that was inoculated (Supplementary Fig. 1).

Identification of resistance loci in Heikezijing. In total, 128 SSR markers with polymorphisms between the two parents were used for constructing the molecular linkage map. This linkage map had a total distance of $1,867.1 \mathrm{cM}$, with an average distance of $14.6 \mathrm{cM}$ between two markers, and covered the whole rice genome according to the information from the IRMI. The average lesion score of each RIL based on the 0 to 5 disease scale was used as the quantitative variable for QTL analysis. When the LOD threshold was set to 3.0, 15 major QTLs corresponding to resistance to 19 blast isolates were detected by the CIM method (Table 2). When the LOD threshold was set to 2.5, five minor Heikezijing QTLs and two minor Suyunuo QTLs were detected (Table 2).

Among the 20 QTLs detected in Heikezijing, 10 QTLs on chromosome 11 corresponded to all tested blast isolates except JS02-95 and JS2004-180, with LOD scores of 3.21 to 38.15 and phenotypic variance of 10.65 to $80.82 \%$, The five QTLs on chromosome 9 were effective against 13 isolates, with LOD scores of 2.65 to 14.57 and phenotypic variance of 7.43 to $68.61 \%$. The four QTLs on chromosome 1 were effective against four isolates, with LOD scores of 2.54 to 2.82, and the one QTL on chromosome 12 was effective against only one isolate, with a LOD score of 6.98 (Table 2). The two minor QTLs from Suyunuo mapped to chromosomes 7 and 11, corresponding to JS02-95 and JS2007-22-2, with 5.04 and $9.91 \%$ of the phenotypic variances, respectively.

TABLE 1. Responses of Heikezijing and Suyunuo to 19 blast isolates

\begin{tabular}{|c|c|c|c|}
\hline \multirow[b]{2}{*}{ Isolate (race) } & \multirow[b]{2}{*}{ Origin } & \multicolumn{2}{|c|}{ Phenotype (lesion score) } \\
\hline & & Heikezijing & Suyunuo \\
\hline GD10-279a(ZB5) & China (Guangdong) & $\mathrm{R}(0)$ & $S(5)$ \\
\hline JS2004-180(ZG1) & China (Jiangsu) & $\mathrm{R}(0)$ & $\mathrm{S}(4.6)$ \\
\hline JS02-68(ZC1) & China (Jiangsu) & $\mathrm{R}(0)$ & $\mathrm{S}(5)$ \\
\hline JS05-74(ZG1) & China (Jiangsu) & $\mathrm{R}(0)$ & $S(5)$ \\
\hline JS02-43(ZD7) & China (Jiangsu) & $\mathrm{R}(0)$ & $\mathrm{S}(5)$ \\
\hline JS90-78(ZF1) & China (Jiangsu) & $\operatorname{MR}(1.3)$ & $\mathrm{S}(5)$ \\
\hline JS2004-185(ZE3) & China (Jiangsu) & $\mathrm{R}(0)$ & $S(5)$ \\
\hline JS2004-183(ZD5) & China (Jiangsu) & $\mathrm{R}(0)$ & $\mathrm{S}(5)$ \\
\hline JS2004-141-1(ZC5) & China (Jiangsu) & $\mathrm{R}(0)$ & $S(5)$ \\
\hline JS02-95(ZE3) & China (Jiangsu) & $\mathrm{R}(0)$ & $\mathrm{S}(5)$ \\
\hline JS03-44(ZA13) & China (Jiangsu) & $\mathrm{R}(0)$ & $S(4.75)$ \\
\hline JS2004-3(ZB5) & China (Jiangsu) & $\operatorname{MR}(1.2)$ & $\mathrm{S}(5)$ \\
\hline JS2007-11-1(ZG1) & China (Jiangsu) & $\mathrm{R}(0)$ & $S(5)$ \\
\hline JS2006-25(ZE3) & China (Jiangsu) & $\mathrm{R}(0)$ & $\mathrm{S}(5)$ \\
\hline JS2007-22-2(ZB29) & China (Jiangsu) & $\mathrm{R}(0)$ & S (4) \\
\hline JS2007-2-3(ZC13) & China (Jiangsu) & $\mathrm{R}(0)$ & $\mathrm{S}(4.1)$ \\
\hline JS2007-51-1(ZD5) & China (Jiangsu) & $\mathrm{R}(0)$ & $\mathrm{S}(5)$ \\
\hline JS2007-2-2(ZF1) & China (Jiangsu) & $\mathrm{R}(0)$ & $\mathrm{S}(4.2)$ \\
\hline Hoku1(ZG1) & Japan & $\mathrm{R}(0)$ & $S(5)$ \\
\hline
\end{tabular}

${ }^{a} \mathrm{R}$ represents resistant, $\mathrm{MR}$ represents moderately resistant, $\mathrm{S}$ represents susceptible, and MS represents moderately susceptible. 
Thirteen QTLs in Heikezijing, including qtll-1-1, qtl1-2-1, qtl1-3-1, qlt1-4-1, qtl9-2-1, qtl9-3-1, qtl9-4-1, qtl9-5-1, qtl11-3-1, qtl11-4-1, qtl11-6-1, qtl11-11-1, and qtl12-1-1, were effective against only one blast isolate and, thus, are more likely to confer isolate-specific resistance. The other seven QTLs may be nonisolate-specific because each QTL confers resistance to more than one isolate, with different peak LOD scores for each isolate (Table 2). For instance, qtl9-1-9 could be detected when inoculated with nine different isolates; qtl11-5-5 and qtl11-7-5 were detected when inoculated with five different isolates; qtl11-10-4 could be detected when inoculated with four different isolates; and qtll1-2-2, qtl11-8-2, and qtll1-9-2 were detected when inoculated with two isolates (Table 2). In addition, eight QTLs detected in Heikezijing contribute to $>30 \%$ of the phenotypic variance, exhibiting a major gene effect. For example, qtl11-5-5 on chromosome 11 could explain 74.34 and $80.82 \%$ of the phenotypic variance when inoculated with Hoku 1 and JS90-78, respectively, and qtl9-4-1 could explain $68.62 \%$ of the phenotypic variance to the blast isolate JS02-95 (Table 2).

Comparison of QTL detection with the expected resistant: susceptible ratio. The resistant:susceptible (R:S) segregation in the RIL population is 1:1 to the isolates JS02-43 and JS2004-3 (Supplementary Table 3), showing a major gene effect. These results are completely consistent with the detection of one QTL. Although the R:S segregation in RIL population is also $1: 1$ to JS2004-183 and JS90-78, two and four QTLs were detected, respectively (Table 2). This could be caused by the united effect of one major QTL and other minor QTLs with lower contributions to phenotypic variance. Like the QTLs conferring resistance to JS90-78, qtl11-5-5 can explain 80.82\% of the phenotypic variance but three other QTLs can only explain 12.28, 45.94, and $25.15 \%$ of the phenotypic variance, respectively.

The R:S segregation in the RIL population is 3:1 to the isolates JS2004-180, JS2007-151-1, JS02-95, JS03-44, Hoku1, JS200625, and JS2007-2-2 (Supplementary Table 3), indicating that the resistance to these isolates is controlled by two major genes. The detection of two QTLs for these isolates fits this model well, except for Hoku1 (Table 2). The R:S segregation to the isolates JS02-68, JS05-74, and JS2007-22-2 is 7:1 (Supplementary Table 3 ), and the detection of three QTLs fits the model well. Although the R:S segregation corresponding to isolates JS2007-11-1, JS2004-185, and JS2007-2-3 were 7:1 or 15:1, two QTLs with lower $R^{2}$ were detected. This suggests that the resistance to these isolate may be controlled by many minor QTLs. In addition, for JS2004-141-1, the R:S segregation is intermediate between 1:1 and 3:1 (Supplementary Table 3), indicating that the resistance is controlled by two genes in which one exhibits a predominant effect. For GD10-279a, the R:S segregation is also intermediate between 1:1 and 3:1. Of the four QTLs were detected, qtl11-10-4 $\left(R^{2}=52.98 \%\right)$ and qtll1-5-5 $\left(R^{2}=32.16 \%\right)$ may play a major role against GD10-279a.

Comparison of the QTLs identified by three approaches. Using the QTLnetwork 2.0 approach, which was developed based on the MCIM (56), only nine resistance QTLs were identified from Heikezijing (Supplementary Table 2). Among the nine QTLs detected, one QTL was detected on chromosome 9 (resistance to 11 isolates), seven QTLs were detected on chromosome 11 (resistance to 18 isolates), and one QTL was detected on chromosome 12 (resistance to 1 isolate). The minor QTLs with LOD between 2.5 and 3.0 by CIM could not be identified by the MCIM approach, whereas the major QTLs (LOD >3.0) corresponding to most isolates could be detected by both approaches, except to the isolate JS2004-180. To JS2004-180, the same QTL on chromosome 9 was detected by both approaches whereas the QTL on chromosome 11 was only detected by MCIM (Table 3). This indicates that the results of QTLnetwork 2.0 can verify the major QTLs detected by CIM from Windows QTL Cartographer 2.5.

Mapmaker/QTL was also used to identify putative loci affecting quantitative resistance, based on point and interval analysis (20). Although Mapmaker/QTL is not designed for recombinant inbred populations, the QTLs could be calculated by changing the data format to $\mathrm{BC}_{1}$ population, as previously described (49). Comparing the results of CIM analysis from Windows QTL Cartographer 2.5, more QTLs were identified based on Mapmaker/QTL analysis (Table 2). The major QTLs on chromosomes 9, 11, and 12 corresponded to the results from the CIM and MCIM methods, and additional minor resistance loci were detected on chromosomes 1, 5, and 7. One QTL (LOD score > 9) on chromosome 4 (between RM335 and RM3367),
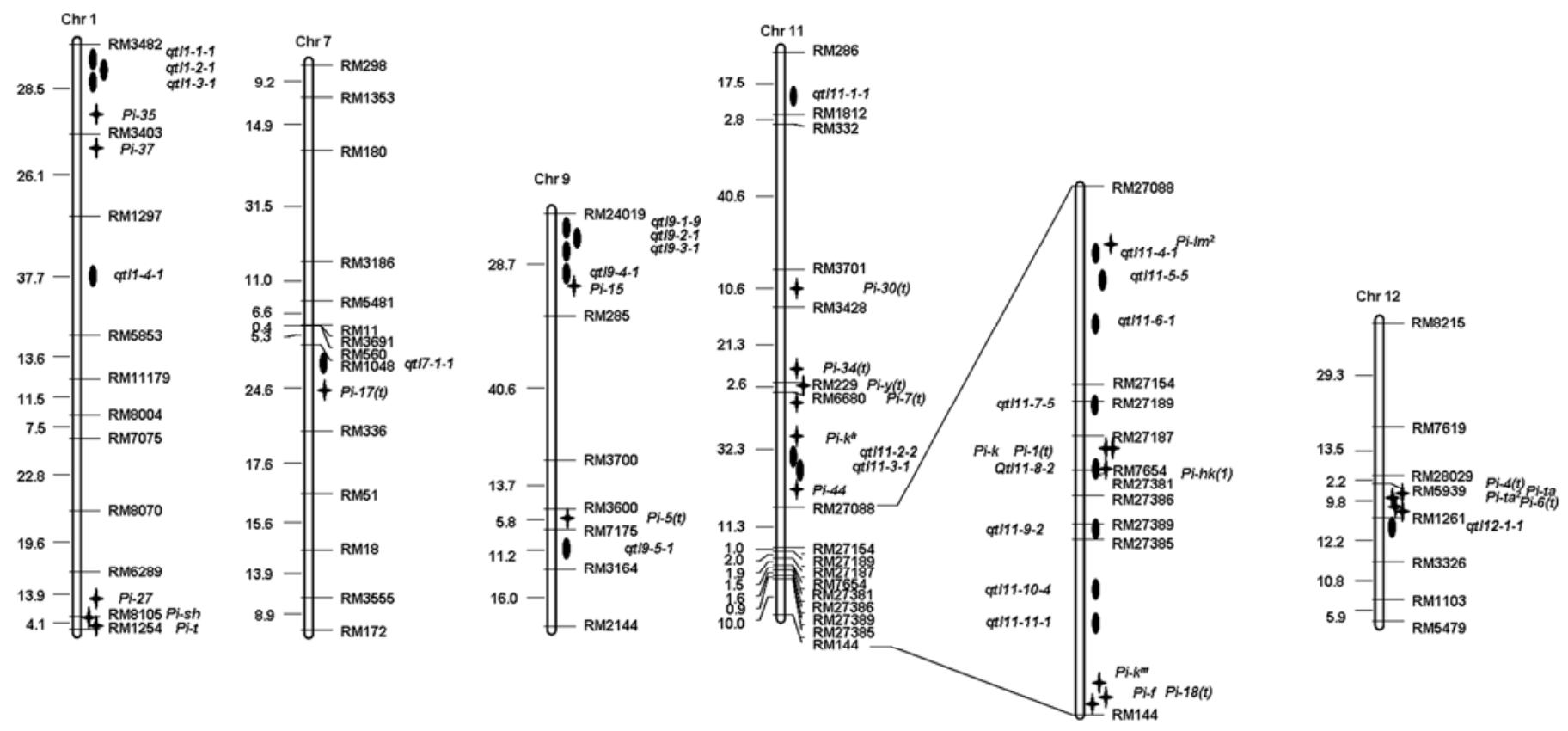

Fig. 1. Genomic locations for quantitative train loci (QTLs) affecting blast resistance of the Heikezijing $\times$ Suyunuo recombinant inbred population. Black ellipses are the QTLs detected by composite interval mapping from Windows QTL Cartographer 2.5 (52); black stars are genes that have been reported previously with an imprecise location on our genetic map. 
corresponding to resistance to JS02-95, JS04-180, JS04-183, JS90-78, and JS2004-3; one QTL on chromosome 10 (between RM333 and RM258), corresponding to resistance to JS2004-3 $(\mathrm{LOD}=6.57)$ and JS2004-141-1 $(\mathrm{LOD}=9.95)$; and one QTL $(\mathrm{LOD}=9.95)$ on chromosome 8 (between RM1019 and RM5853), corresponding to resistance to JS2004-141-1 (Table 3) were detected. None of these QTLs were detected by the CIM and MCIM methods (Table 2). Although these QTLs may be major genes according to their LOD scores and $R^{2}$ (Supplementary Table 1), it was found that these markers did not correlate with the lesion scores of the corresponding isolates after co-segregation analysis (data not shown). Some pseudo-QTLs may be detected by Mapmaker/QTL due to accumulated $R^{2}$ of QTLs to one isolate being far more than $100 \%$ (Supplementary Table 1).

\section{DISCUSSION}

The japonica rice landrace Heikezijing was resistant to all 19 isolates of $M$. grisea tested in this study, indicating that this landrace is a typical first-type broad-resistant accession (41).
Usually, the rice resistance to blast is thought of as a qualitative trait when the reaction to blast is scored on a six-class scale based on lesion types (43). In this system, the plants with scores of 0,1 , and 2 are referred to as resistant and those with scores of 3, 4, and 5 are referred to as susceptible. Some plants with critical scores of 2 or 3 are difficult to clearly classify as resistant or susceptible; therefore, it is necessary to further analyze their resistance or susceptibility using segregation in the $F_{2}$ and $F_{3}$ generation $(1,8$, $9,26,30,39)$. To effectively dissect Heikezijing resistance genes to various isolates of blast, a population of 166 RILs $\left(\mathrm{F}_{2: 8}\right)$ was produced from a cross between Heikezijing and a highly blastsusceptible japonica cultivar, Suyunuo. Through the population of RILs derived from the resistant $\times$ susceptible accession, the reaction of each line to one blast isolate could be accurately evaluated using an average score from a number of individuals.

The QTL detection approach has been widely used in order to localize major or minor loci involved in resistance at the molecular level $(13,43,45)$. Our results found a number of QTLs in Heikezijing, including several major QTLs on chromosomes 9 and 11 which conferred resistance to most of the isolates, one

TABLE 2. Quantitative train loci (QTLs) for resistance to blast detected based on lesion scores

\begin{tabular}{|c|c|c|c|c|c|c|c|c|}
\hline Isolates (race) & $\mathrm{QTLs}^{\mathrm{a}}$ & Chr. no. ${ }^{b}$ & Position $(\mathrm{cM})^{\mathrm{c}}$ & $\mathrm{LOD}^{\mathrm{d}}$ & Additive $^{\mathrm{e}}$ & $R^{2}(\%)^{\mathrm{f}}$ & Marker left & Marker right \\
\hline \multirow[t]{2}{*}{ JS2004-180(ZG1) } & qtl1-1-1* & 1 & 0.01 & 2.61 & -0.3621 & 4.4494 & RM3482 & RM3403 \\
\hline & qtl9-1-9 & 9 & 0.01 & 9.25 & -0.716 & 17.3416 & RM24019 & RM285 \\
\hline \multirow[t]{2}{*}{ JS2007-51-1(ZD5) } & qtl9-5-1* & 9 & 96.81 & 2.83 & -0.3352 & 7.4953 & RM7175 & RM2144 \\
\hline & qtl11-8-2 & 11 & 143.81 & 11.43 & -0.6827 & 31.3272 & RM27187 & RM27389 \\
\hline \multirow[t]{2}{*}{ JS2004-183(ZD5) } & qtl11-10-4 & 11 & 149.81 & 15.38 & -1.115 & 34.1111 & RM27389 & RM144 \\
\hline & qtl12-1-1 & 12 & 56.81 & 6.98 & -0.6809 & 12.3970 & RM28089 & RM3326 \\
\hline \multirow[t]{3}{*}{ JS02-68(ZC1) } & qtl9-1-9 & 9 & 0.01 & 6.2 & -0.4647 & 12.9744 & RM24019 & RM285 \\
\hline & qtll1-4-1 & 11 & 131.61 & 4.5 & -0.5378 & 12.4631 & RM27088 & RM27154 \\
\hline & qtl11-10-4 & 11 & 149.81 & 9.81 & -0.685 & 25.9149 & RM27386 & RM144 \\
\hline \multirow[t]{3}{*}{ JS05-74(ZG1) } & qtll-2-1* & 1 & 6.01 & 2.69 & -0.3 & 7.8450 & RM3482 & RM3403 \\
\hline & qtl9-1-9 & 9 & 0.01 & 3.76 & -0.2965 & 7.4278 & RM24019 & RM285 \\
\hline & qtl11-7-5 & 11 & 139.91 & 8.92 & -0.4998 & 19.4588 & RM27154 & RM27381 \\
\hline \multirow[t]{2}{*}{ JS2007-2-3(ZC13) } & qtl9-1-9* & 9 & 0.01 & 2.65 & -0.2231 & 7.9592 & RM24019 & RM285 \\
\hline & qtl11-7-5 & 11 & 139.91 & 3.21 & -0.2701 & 10.6505 & RM27088 & RM27187 \\
\hline \multirow[t]{2}{*}{ JS02-95(ZE3) } & qtl7-1-1* & 7 & 78.91 & 2.54 & 0.4027 & 5.0494 & RM11 & RM336 \\
\hline & qtl9-4-1 & 9 & 14.01 & 14.57 & -1.7814 & 68.6169 & RM24019 & RM285 \\
\hline \multirow[t]{2}{*}{ JS2007-11-1(ZG1) } & qtl9-1-9 & 9 & 0.01 & 4.38 & -0.4246 & 10.0918 & RM24019 & RM285 \\
\hline & qtl11-6-1 & 11 & 135.61 & 5.75 & -0.5957 & 17.9911 & RM27088 & RM27154 \\
\hline JS02-43(ZD7) & qtl11-9-2 & 11 & 146.91 & 15.65 & -1.1933 & 42.8057 & RM27386 & RM144 \\
\hline \multirow[t]{2}{*}{ JS03-44(ZA13) } & qtl9-3-1 & 9 & 4.01 & 5.07 & -0.6146 & 14.2235 & RM24019 & RM285 \\
\hline & qtl11-9-2 & 11 & 146.91 & 5.75 & -0.5904 & 13.2858 & RM27386 & RM144 \\
\hline \multirow[t]{4}{*}{ JS90-78(ZF1) } & qtl1-4-1* & 1 & 72.61 & 2.54 & -0.7186 & 12.2871 & RM1297 & RM5853 \\
\hline & qtl11-2-2 & 11 & 115.31 & 14.95 & -1.7172 & 45.9418 & RM6680 & RM27088 \\
\hline & qtl11-5-5 & 11 & 133.61 & 20.44 & -1.8804 & 80.8162 & RM27088 & RM27154 \\
\hline & qtl11-10-4 & 11 & 149.81 & 8.03 & -1.1887 & 25.1497 & RM27389 & RM144 \\
\hline JS2004-3(ZB5) & qtl11-8-2 & 11 & 143.81 & 9.19 & -0.8612 & 23.3719 & RM27189 & RM27389 \\
\hline \multirow[t]{2}{*}{ JS2004-141-1(ZC5) } & qtl11-2-2 & 11 & 115.31 & 5.47 & -1.4067 & 39.3976 & RM6680 & RM27088 \\
\hline & qtl11-5-5 & 11 & 133.61 & 9.93 & -1.0887 & 38.3425 & RM27088 & RM27154 \\
\hline \multirow[t]{3}{*}{ JS2007-22-2(ZB29) } & $q t l 9-1-9$ & 9 & 0.01 & 5.3 & -0.4232 & 15.3173 & RM24019 & RM285 \\
\hline & qtl11-1-1* & 11 & 14.01 & 2.7 & 0.3399 & 9.9050 & RM286 & RM1812 \\
\hline & qtl11-7-5 & 11 & 139.91 & 4.6 & -0.4087 & 13.3469 & RM27154 & RM27381 \\
\hline \multirow[t]{3}{*}{ Hoku1(ZG1) } & qtl11-3-1 & 11 & 119.31 & 13.98 & -1.5197 & 33.5064 & RM6680 & RM27088 \\
\hline & qtll1-5-5 & 11 & 133.61 & 38.15 & -1.6211 & 74.3417 & RM27088 & RM27154 \\
\hline & qtl11-11-1 & 11 & 151.81 & 17.55 & -1.5763 & 47.3126 & RM27385 & RM144 \\
\hline \multirow[t]{2}{*}{ JS2006-25(ZE3) } & $q t l 9-1-9$ & 9 & 0.01 & 6.9 & -0.5819 & 13.2528 & RM24019 & RM285 \\
\hline & qtl11-7-5 & 11 & 139.91 & 9.57 & -0.7456 & 19.9360 & RM27154 & RM27381 \\
\hline \multirow[t]{2}{*}{ JS2004-185(ZE3) } & qtl9-1-9 & 9 & 0.01 & 4.76 & -0.4364 & 9.5439 & RM24019 & RM285 \\
\hline & qtl11-5-5 & 11 & 133.61 & 7.9 & -0.6905 & 21.6825 & RM27088 & RM27154 \\
\hline \multirow[t]{4}{*}{ GD10-279a(ZB5) } & qtll-3-1* & 1 & 8.01 & 2.82 & -0.4386 & 6.0319 & RM3482 & RM3403 \\
\hline & qtl9-2-1 & 9 & 2.01 & 8.62 & -0.6878 & 15.1849 & RM24019 & RM285 \\
\hline & qtl11-5-5 & 11 & 133.61 & 9.8 & -1.3341 & 32.1659 & RM27088 & RM27154 \\
\hline & qtl11-10-4 & 11 & 149.81 & 23.3 & -1.3505 & 52.9777 & RM27385 & RM144 \\
\hline \multirow[t]{2}{*}{ JS2007-2-2(ZF1) } & qtl9-1-9 & 9 & 0.01 & 4.6 & -0.5112 & 11.8846 & RM24019 & RM285 \\
\hline & qtl11-7-5 & 11 & 139.91 & 7.03 & -0.686 & 19.6855 & RM27189 & RM27381 \\
\hline
\end{tabular}

a QTLs with “*” were detected when logarithm of the odds (LOD) threshold down to 2.5.

b Chromosome number.

c Peak LOD score in the QTL.

d Position in centimorgans (cM) of peak LOD score.

e Additive effect explained at peak LOD score.

${ }^{\mathrm{f}}$ Explained the contribution to the phenotypic variance. 
QTL on chromosome 12 which was effective against the isolate JS2004-183, and a number of minor loci on chromosomes 1 and 9 (Fig. 1). In general, the $R$ genes fall into six distinct classes, the most prevalent of which encodes an NBS and a series of carboxyterminal LRRs (33). In all, $\approx 600$ NBS-LRR genes have been identified in the rice genome but $>100$ of these were predicted to be pseudogenes in rice cv. Nipponbare $(3,37)$. Most putative $R$ genes are present in clusters. Rice chromosome 11 is rich in disease-resistance genes and has at least 201 putative $R$ genes $(42,62)$. In fact, it has been reported that there are many genes resistant to blast that are located on the long arm of chromosome 11 (Fig. 1), such as Pil(t) (31), Pik (34), Pi-lm2 (46), Pi-18 (1), Pi44(t) (8), Pi-30(t) (43), Pi34 (57,58), Pi-y(t) (60), Pik-h (44), $P i f$, and Pi7 (12). Recently, Pik-m and Pi-hkl(t) were reported to be localized in this region $(22,23)$.

The QTLs in this study were mapped to the chromosomal locations where other blast resistance genes have been located, except for two minor QTLs (qtll-4-1 and qtl9-5-1) (Fig. 1). The QTLs may correspond to the specific resistance genes already described at these map positions or to different alleles. However, allelism tests between reference cultivars with known resistance genes will be necessary to demonstrate whether these QTLs are new resistance gene alleles. Twenty QTLs were detected that confer resistance to 19 blast isolates (Table 2). A cluster of 10 blast resistance loci on chromosome 11 was detected between SSR markers RM6680 and RM144, close to a series of blast resistance genes (Fig. 1) The QTLs effective against more isolates, such as qtl11-2-2 (resistant to two isolates), qtl11-5-5 (resistant to five isolates), and qtl11-7-5 (resistant to five isolates), may confer the broad-spectrum resistance. However, none of these loci were effective against all 19 isolates tested in this study. It can be concluded that there is an $R$-gene cluster found on the long arm of chromosome 11 in the japonica landrace Heikezijing, and QTLs detected here likely function to confer race-specific resistance.

Qtl1-1-1, qtl1-2-1, and qtl1-3-1, identified on chromosome 1, mapped closely to the blast resistance genes Pi35(t) and Pi37 (9,38). Qtt9-1-9, qtl9-2-1, qtl9-3-1, and qtl9-4-1, located on chromosome 9, are close to the blast resistance genes Pi5 and Pil5 (16,39). Qtl12-1-1 located on chromosome 12 mapped closely to a blast resistance gene cluster including $P i$-ta(6). The QTLs on chromosomes 1 and 9 were also distributed in a cluster (Fig. 1). The QTLs on chromosome 1 were detected with a lower LOD threshold, suggesting that they may confer partial resistance to blast. Four QTLs on chromosome 9 correspond to the resistance to 12 isolates and qtl9-1-9 corresponds to 9 isolates, suggesting that qtl9-1-9 may be a broad-spectrum resistance gene.

Through comparing the results of three analysis approaches, we found that Mapmaker/QTL could detect more QTLs, the QTL network 2.0 based on MCIM could detect most of the major QTLs, and WinQTLCartgrapher 2.5 based on CIM could detect both major QTLs and the minor loci. The QTLs estimated by the CIM method were used for further analysis. Two QTLs from the susceptible parent Suyunuo, corresponding to resistance to JS02-

TABLE 3. Comparison of the results of different analysis approaches

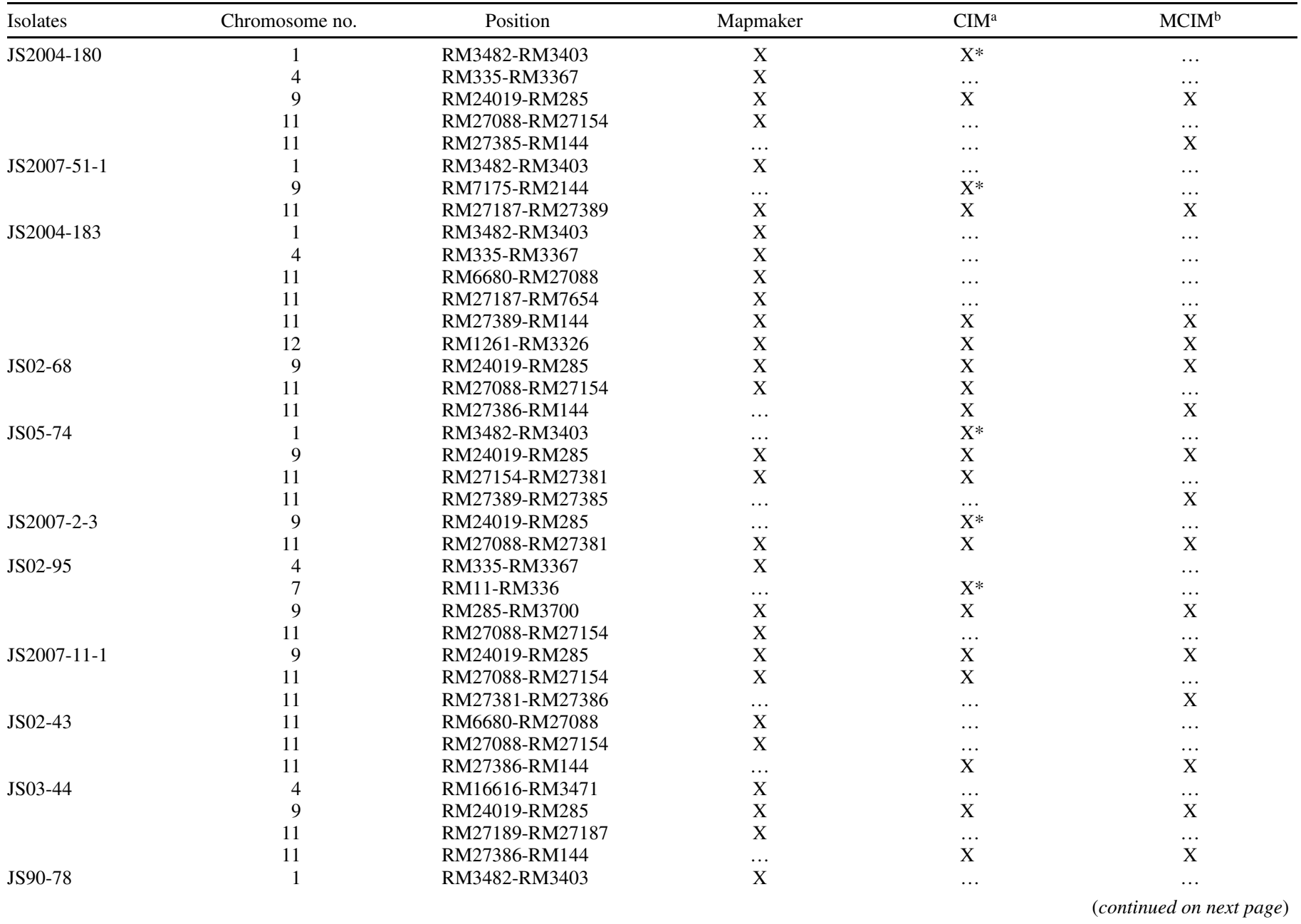

a Asterisk (*) indicates the minor quantitative train loci detected by the composite interval mapping (CIM) of Windows QTL Cartographer 2.5 (52).

${ }^{b}$ Mixed-model-based CIM. 
95 and JS2007-22-2, were detected on chromosome 7 (LOD score $=2.54)$ and chromosome 11 (LOD score $=2.70)$, respectively (Table 2), implying that minor genes conferring blast resistance might exist in Suyunuo.

In addition, the results of QTL analysis do not correlate with the class of isolates based on the Chinese rice blast identification system (25). Some QTLs, such as qtl11-5-5, could be responsible for the resistance to multiple isolates, including B group (GD10279a), C group (JS2004-141-1), E group (JS2004-185), F group (JS90-78), and G group (Hoku 1), but the resistance to isolates in the same group did not correspond to the same QTLs detected here (Table 2).

Many resistance genes have been mapped as a qualitative trait by using bulked-segregate analysis (BSA) in order to find tightly linked molecular markers (35). Through the BSA approach, several genes resistant to blast-Pi5(t), Pi36, and Pi27(t) - were mapped onto chromosomes 8,1 , and 9 , respectively $(16,29,63)$. Recently, a new rice blast resistance gene, Pi40, was mapped onto chromosome 6 using the single-locus analysis of variance (ANOVA) test (17). QTL identification using the CIM approach, based on genetic a linkage map, can detect more than one gene and not only major loci but also minor loci associated with resistance (43). Li et al. (23) identified the resistance gene $\mathrm{Pi}$ $h k l(t)$ from Heikezijing using the BSA method (36). With 166 RILs and the CIM approach used in this study, three major tightly linked QTLs to Hoku 1 were detected, with LOD scores of 13.98, 38.15 , and 17.55 and $33.5,74.3$, and $47.3 \%$ of the phenotypic variance, respectively (Table 2). Thus, the major QTL (qtl11-5-5) with the highest LOD score might be Pi-hkl(t) identified by BSA (23). BSA may be considered suitable for finding a single pathogen resistance gene in a plant but it difficult to find genelinked markers when there are two or more major genes corresponding to resistance to the same pathogen or when minor loci near the single gene could affect linkage analysis.

The frequency of polymorphisms between Heikezijing and Suyunuo was not as high as for the indicaljaponica crosses. There are some crossover-cold regions that exist in this linkage map. One strategy to clone resistance genes in Heikezijing is to choose a line with a single resistant locus from RILs and cross with a susceptible indica cultivar.

In summary, we mapped rice blast resistance QTLs using RILs from the cross of Heikezijing $\times$ Suyunuo. The results showed that several QTLs with various contribution rates were responsible for the blast resistance in Heikezijing. In particular, one blast-resistance-gene cluster was found on chromosome 11. It will be valuable to further characterize and clone genes resistant to blast in this japonica landrace in order to breed new rice cultivars with resistance to blast using genetic engineering or molecular assistance selection approaches.

\section{ACKNOWLEDGMENTS}

This research was supported by grants from the National Key Project for Transgenic Crops (2008ZX08009-003 and 2009ZX08009-040B), the

TABLE 3. (continued from preceding page)

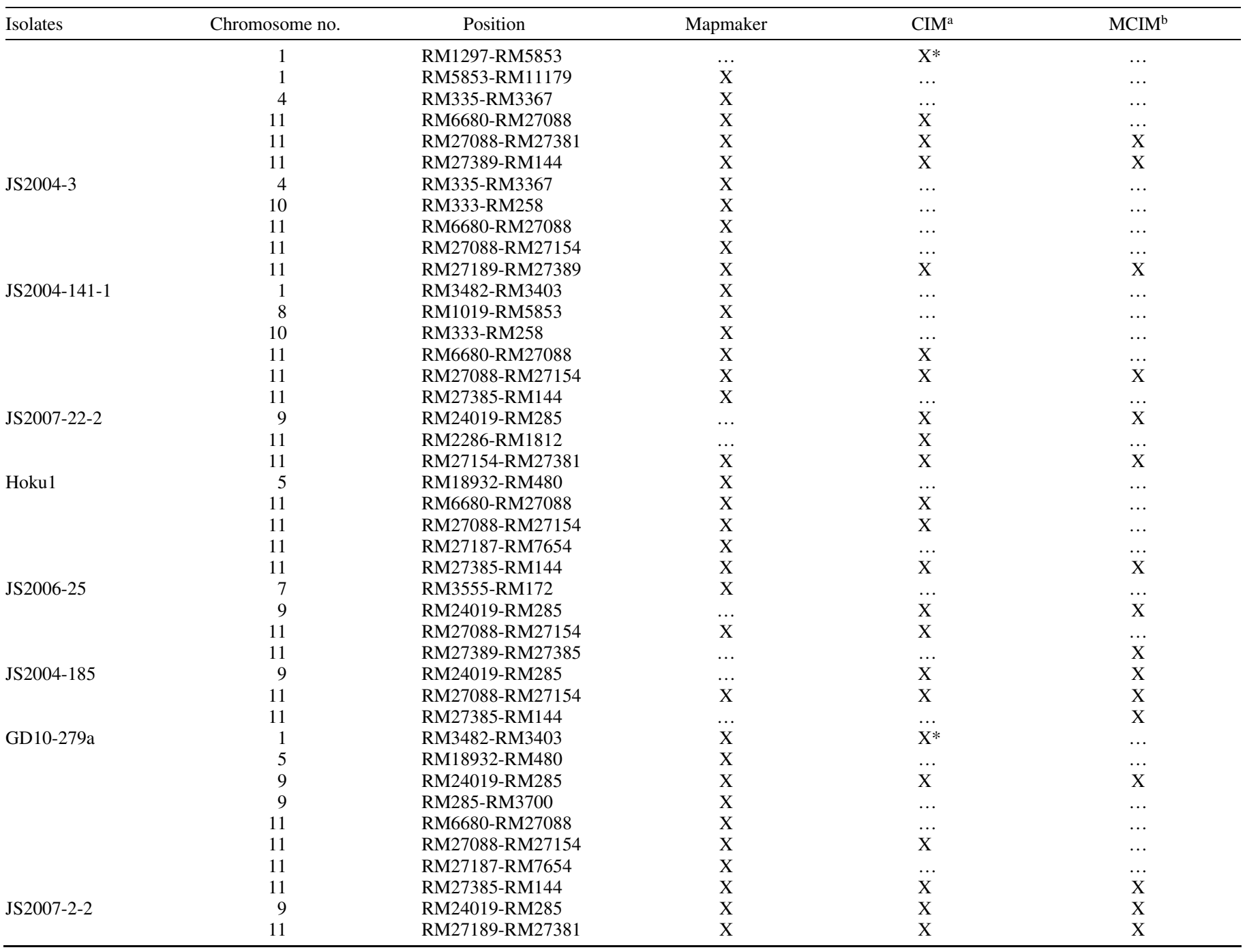


Natural Science Foundation of China (30771324), Natural Science Foundation of Jiangsu province (BK2009306), and National "863" Project (2006AA10Z162). We thank Z.-Y. Chen and Z.-Z. Ling for kindly providing the blast isolates and Y.-F. Liu for experimental help.

\section{LITERATURE CITED}

1. Ahn, S. N., Kim, Y. K., Hong, H. C., Han, S. S., Kwon, S. J., Choi, H. C., Moon, H. P., and McCouch, S. R. 2000. Molecular mapping of a new gene for resistance to rice blast (Pyricularia grisea Sacc.). Euphytica 116:17-22.

2. Ashikawa, I., Hayashi, N., Yamane, H., Kanamori, H., Wu, J., Matsumoto, T. , Ono, K., and Yano, M. 2008. Two adjacent nucleotidebinding site-leucine-rich repeat class genes are required to confer Pikmspecific rice blast resistance. Genetics 180:2267-2276.

3. Bai, J. F., Pennill, L. A., Ning, J. C., Lee, S. W., Ramalingam, J., Webb, C. A., Zhao, B. Y., Sun, Q., Nelson, J. C., Leach, J. E., and Hulbert, S. H. 2002. Diversity in nucleotide binding site-leucine-rich repeat genes in cereals. Genome Res. 12:1871-1884

4. Berruyer, R., Adreit, H., Milazzo, J., Gaillard, S., Berger, A., Dioh, W., Lebrun, M. H., and Tharreau, D. 2003. Identification and fine mapping of Pi33, the rice resistance gene corresponding to the Magnaporthe grisea avirulence gene ACE1. Theor. Appl. Genet. 107:1139-1147.

5. Bonman, M. J., Khush, G. S., and Nelson, R. J. 1992. Breeding rice for resistance to pests. Annu. Rev. Phytopathol. 30:507-528.

6. Bryan, G. T., Wu, K.S., Farrall, L., Jia, Y., Hershey, H. P., McAdams, S. A., Faulk, K. N., Donaldson, G. K., Tarchini, R., and Valent, B. 2000. A single amino acid difference distinguishes resistant and susceptible alleles of the rice blast resistance gene Pi-ta. Plant Cell 12:2033-2046.

7. Buschges, R., Hollricher, K., Panstruga, R., Simons, G., Wolter, M., Frijters, A., Daelen, R., Lee, T., Diergaarde, P., Groenendijk, J., Topsch, S., Vos, P., Salamini, F., and Paul, S. L. 1997. The barley Mlo gene: A novel control element of plant pathogen resistance. Cell 88:695-705.

8. Chen, D. H., Vina, M., Inukai, T., Mackil, D. J., Ronald, P. C., and Nelson, R. J. 1999. Molecular mapping of the blast resistance gene, Pi44(t), in a line derived from a durably resistant rice cultivar. Theor. Appl. Genet. 98:1046-1053.

9. Chen, S., Wang, L., Que, Z. Q., Pan, R. Q., and Pan, Q. H. 2005. Genetic and physical mapping of $P i 37(t)$, a new gene conferring resistance to rice blast in the famous cultivar St. No. 1. Theor. Appl. Genet. 111:15631570 .

10. Chen, X. W., Shang, J. J., Chen, D. X., Lei, C. L., Zou, Y., Zhai, W. X., Liu, G. Z., Xu, J. C., Ling, Z. Z., Cao, G., Ma, B. T., Wang, Y. P., Zhao, X. F., Li, S. G., and Zhu, L. H. 2006. A B-lectin receptor kinase gene conferring rice blast resistance. Plant J. 46:794-804

11. Deng, Y. W., Zhu, X. D., Shen, Y., and He, Z. H. 2006. Genetic characterization and fine mapping of the blast resistance locus Pigm $(t)$ tightly linked to $\mathrm{Pi2}$ and $\mathrm{Pi} 9$ in a broad-spectrum resistant Chinese variety. Theor. Appl. Genet. 113:705-713.

12. Fuentes, J. L., Correa-Victoria, J. F., Escobar, F., Prado, G., Aricapa, G., Duque, M. C., and Tohme, J. 2008. Identification of microsatellite markers linked to the blast resistance gene $P i-1(t)$ in rice. Euphytica 160:295-304.

13. Fukuoka, S., and Okuno, K. 2001. QTL analysis and mapping of pi21, a recessive gene for field resistance to rice blast in Japanese upland rice. Theor. Appl. Genet. 103:185-190.

14. Fukuoka, S., Saka, N., Koga, H., Ono, K., Shimizu, T., Ebana, K., Hayashi, N., Takahashi, A., Hirochika, H., Okuno, K., and Yano, M. 2009. Loss of function of a proline-containing protein confers durable disease resistance in rice. Science 325:998-1001.

15. Hayashi, K., and Yoshida, H. 2009. Refunctionalization of the ancient rice blast disease resistance gene Pit by the recruitment of a retrotransposon as a promoter. Plant J. 57:413-425.

16. Jeon, J. S., Chen, D., Yi, G. H., Wang, G. L., and Ronald, P. C. 2003. Genetic and physical mapping of Pi5 $(t)$, a locus associated with broadspectrum resistance to rice blast. Mol. Genet. Genomics 269:280-289.

17. Jeung, J. U., Kim, B. R., Cho, Y. C., Han, S. S., Moon, H. P., Lee, Y. T., and Jena, K. K. 2007. A novel gene, Pi40(t), linked to the DNA markers derived from NBS-LRR motifs confers broad spectrum of blast resistance in rice. Theor. Appl. Genet. 115:1163-1177.

18. Kosambi, D. 1944. The estimation of map distance from recombination values. Ann. Eugen. 12:172-175.

19. Lander, E., and Kruglyak, L. 1995. Genetic dissection of complex traits: guidelines for interpreting and reporting linkage results. Nat. Genet. 11:241-247.

20. Lander, E. S., Green, P., Abrahamson, J., Barlow, A., Daly, M. J., Lincoln, S. E., and Newburg, L. 1987. MAPMAKER: an interactive computer package for constructing primary genetic linkage maps of experimental and natural populations. Genomics 1:174-181.
21. Lee, S. K., Song, M. Y., Seo, Y. S., Kim, H. K., Ko, S., Cao, P. J., Suh, J. P., Yi, G., Roh, J. H., Lee, S., An, G., Hahn, T. R., Wang, G. L., Ronald, P., and Jeon, J. S. 2009. Rice Pi5-mediated resistance to Magnaporthe oryzae requires the presence of two coiled-coil-nucleotide-bindingleucine-rich repeat genes. Genetics 181:1627-1638.

22. Li, L. Y., Wang, L., Jing, J. X., Li, Z. Q., Lin, F., Huang, L. F., and Pan, Q. H. 2007. The Pikm gene, conferring stable resistance to isolates of Magnaporthe oryzae, was finely mapped in a crossover-cold region on rice chromosome 11. Mol. Breed. 20:179-188.

23. Li, P. F., Shi, X. L., Wang, J. F., Liu, C., and Zhang, H. S. 2007. Molecular mapping of rice blast resistance gene in a japonica landrace Heikezijing from the Taihu Lake area, China. Chin. J. Rice Sci. 21:579584.

24. Lin, F., Chen, S., Que, Z. Q., Wang, L., Liu, X. Q., and Pan, Q. H. 2007. The blast resistance gene Pi37 encodes a nucleotide binding site-leucinerich repeat protein and is a member of a resistance gene cluster on rice chromosome1. Genetics 177:1871-1880.

25. Ling, Z. H., Lei, C. L., and Wang, J. L. 2004. Review and prospect for study of physiologic races on rice blast fungus (Pyricularia grisea). Sci. Agric. Sin. 37:1849-1859.

26. Liu, G., Lu, G., Zeng, L., and Wang, G.L. 2002. Two broad-spectrum blast resistance genes, $P i 9(t)$ and $P i 2(t)$, are physically linked on rice chromosome 6. Mol. Genet. Genomics 267:472-480.

27. Liu, R. H., and Meng, J. L. 2003. MapDraw: a Microsoft Excel macro for drawing genetic linkage maps based on given genetic linkage data. Hereditas (Beijing) 25:317-321

28. Liu, X. Q., Lin, F., Wang, L., and Pan, Q. H. 2007. The in silico mapbased cloning of Pi36, a rice coiled-coil nucleotide-binding site leucinerich repeat gene that confers race-specific resistance to the blast fungus. Genetics 176:2541-2549.

29. Liu, X. Q., Wang, L., Chen, S., Lin, F., and Pan, Q. H. 2005. Genetic and physical mapping of $P i 36(t)$, a novel rice blast resistance gene located on rice chromosome 8. Mol. Genet. Genomics 274:394-401.

30. Liu, X. Q., Yang, Q. Z., Lin, F., Hua, L. X., Wang, C. T., Wang, L., and Pan, Q. H. 2007. Identification and fine mapping of Pi39(t), a major gene conferring the broad-spectrum resistance to Magnaporthe oryzae. Mol. Genet. Genomics 278:403-410.

31. Mackill, D., and Bonman, J. 1992. Inheritance of blast resistance in nearisogenic lines of rice. Phytopathology 82:746-749.

32. Manosalva, P. M., Davidson, R. M., Liu, B., Zhu, X. Y., Hulbert, S. H., Leung, H., and Leach, J. E. 2009. A germin-like protein gene family functions as a complex quantitative trait locus conferring broad-spectrum disease resistance in rice. Plant Physiol. 149:286-269.

33. Martin, G. B., Bogdanove, A. J., and Sessa, G. 2003. Understanding the functions of plant disease resistance proteins. Annu. Rev. Plant Biol. 54:23-61.

34. McCouch, S. R., Nelson, R. J., Thome, J., and Zeigler, R. S. 1994. Mapping of blast-resistance genes in rice. Pages 167-187 in: Rice Blast Disease. R. S. Zeigler, S. A. Leong, and P. S. Teng, eds. CAB International, Wallingford, UK.

35. Mew, T. V., Parco, A. S., Hittalmani, S., Inukai, T., and Nelson, R. 1994. Fine-mapping of major genes for blast resistance in rice. Rice Genet. Newsl. 11:126-128.

36. Michelmore, R., Paranand, I., and Kessali, R. 1991. Identification of markers linked to disease resistance gene by bulked segregant analysis: a rapid method to detect markers in specific genomic region using segregating populations. Proc. Natl. Acad. Sci. USA 88:9829-9832.

37. Monosi, B., Wisser, R. J., Pennill, L., and Hulbert, S. H. 2004. Fullgenome analysis of resistance gene homologues in rice. Theor. Appl. Genet. 109:1434-1447.

38. Nguyen, T. T. T., Koizumi, S., La, T. N., Zenbayshi, K. S., Ashizawa, T., Yasuda, N., Imazaki, I., and Miyasaka, A. 2006. Pi35(t), a new gene conferring partial resistance to leaf blast in the rice cultivar Hokkai 188. Theor. Appl. Genet. 113:697-704.

39. Pan, Q. H., Hu, Z. D., Takatoshi, T., and Wang, L. 2003. Fine mapping of the blast resistance gene Pi15, linked to Pii, on rice chromosome 9. Acta Bot. Sin. 45:871-877.

40. Pich, U., and Schubert, I. 1993. Midiprep method for isolation of DNA from plants with a high content of polyphenolics. Nucleic Acids Res. 21:3328-3332.

41. Qu, S. H., Liu, G. F., Zhou, B., Bellizzi, M., Zeng, L. R., Dai, L. Y., Han, B., and Wang, G. L. 2006. The broad-spectrum blast resistance gene Pi9 encodes a nucleotide-binding site-leucine-rich repeat protein and is a member of a multigene family in rice. Genetics 172:1901-1914.

42. Rice Chromosomes 11 and 12 Sequencing Consortia. 2005. The sequence of rice chromosomes 11 and 12, rich in disease resistance genes and recent gene duplications. BMC Biol. 3:20-37.

43. Sallaud, C., Lorieux, M., Roumen, E., Tharreau, D., Berruyer, R., Svestasrani, P., Garsmeur, O., Ghesquiere, A., and Notteghem, J. L. 2003. Identification of five new blast resistance genes in the highly blast- 
resistant rice variety IR64 using a QTL mapping strategy. Theor. Appl. Genet. 106:794-803.

44. Sharma, T. R., Madhav, M. S., Singh, B. K., Shanker, P., Jana, T. K., Dalal, V., Pandit, A., Singh, A., Gaikwad, K., Upreti, H. C., and Singh, N. K. 2005. High-resolution mapping, cloning and molecular characterization of the $P i-k^{h}$ gene of rice, which confers resistance to Magnaporthe grisea. Mol. Genet. Genomics 274:569-578.

45. Sirithunya, P., Tragoonrung, S., Vanavichit, A., Pa-In, N., Vongsaprom, C., and Toojinda, T. 2002. Quantitative trait loci associated with leaf and neck blast resistance in recombinant inbred line population of rice (Oryza sativa). DNA Res. 9:79-88.

46. Tabien, R. E., Li, Z., Paterson, A. H., Marchetti, M. A., Stansel, J. W., and Pinson, S. R. M. 2000. Mapping of four major rice blast resistance genes from 'Lemont' and 'Teqing' and evaluation of their combinatorial effect for field resistance. Theor. Appl. Genet. 101:1215-1225.

47. Tabien, R. E., Li, Z., Paterson, H., Marchetti, A., Stansel, W., and Pinson, M. 2002. Mapping QTLs for field resistance to the rice blast pathogen and evaluating their individual and combined utility in improved varieties. Theor. Appl. Genet. 105:313-324.

48. Valent, B., and Chumley, F. G. 1991. Molecular genetic analysis of the rice blast fungus, Magnaporthe grisea. Annu. Rev. Phytopathol. 29:443467.

49. Wang, G. L., Mackill, D. J., Bonman, J. M., McCouch, S. R., Champoux, M. C., and Nelson, R. J. 1994. RFLP mapping of genes conferring complete and partial resistance to blast in a durably resistant rice cultivar. Genetics 136:1421-1434.

50. Wang, G. L., Song, W. Y., Ruan, D. L., Sideris, S., and Ronald, P. C. 1996. The closed gene, Xa21, confers resistance to multiple Xanthomonas oryzae pv. oryzae isolates in transgenic plants. Mol. Plant-Microbe Interact. 9:850-855.

51. Wang, J. F., He, X. J., Zhang, H. S., and Chen, Z. Y. 2002. Genetic analysis of blast resistance in japonica rice landrace Heikezijing from Taihu Region. Acta Genet. Sin. 29:803-807.

52. Wang, S., Basten, C. J., and Zeng, Z. B. 2007. Windows QTL Cartographer 2.5. Department of Statistics, North Carolina State University, Raleigh. http://statgen.ncsu.edu/qtlcart/WQTLCart.htm.

53. Wang, Z. X., Yano, M., Yamanouchi, U., Iwamoto, M., Monna, L., Hayasaka, H., Katayose, Y., and Sasaki, T. 1999. The Pib gene for rice blast resistance belongs to the nucleotide binding and leucine-rich repeat class of plant disease resistance genes. Plant J. 19:55-64.

54. Wu, J. L., Fan, Y. Y., Li, D. B., Zheng, K. L., Leung, H., and Zhuang, J. Y. 2005. Genetic control of rice blast resistance in the durably resistant cultivar Gumei 2 against multiple isolates. Theor. Appl. Genet. 111:5056.

55. Xiao, S., Ellwood, S., Calis, O., Patrick, E., Li, T., Coleman, M., and Turner, J. G. 2001. Broad-spectrum mildew resistance in Arabidopsis thaliana mediated by RPW8. Science 291:118-120.

56. Yang, J., Hu, C. C., Ye, X. Z., and Zhu, J. 2005. QTLNetwork 2.0.Institute of Bioinformatics, Zhejiang University, Hangzhou, China. http://ibi.zju.edu.cn/software/qtlnetwork.

57. Zenbayashi-Sawata, K., Ashizawa, T., and Koizumi, S. 2005. Pi34AVRPi34: a new gene-for-gene interaction for partial resistance in rice to blast caused by Magnaporthe grisea. J. Gen. Plant Pathol. 71:395-401.

58. Zenbayashi-Sawata, K., Fukuoka, S., Katagiri, S., Fujisawa, M., Matsumoto, T., Ashizawa, T., and Koizumi, S. 2007. Genetic and physical mapping of the partial resistance gene Pi34, to blast in rice. Phytopathology 97:598-602.

59. Zeng, Z. B. 1994. Precision mapping of quantitative trait loci. Genetics $136: 1457-1468$

60. 60 Zhang, J. F., Ling, Z. Z., Wang, G. Y., and Xie, H. A. 2006. Mapping blast-resistance genes of japonica rice Yunyin by SSR markers. Mol. Plant Breed. 4:359-364.

61. Zhou, B., Qu, S. H., Liu, G. F., Dolan, M., Sakai, H., Lu, G. D., Bellizzi, M., and Wang, G. L. 2006. The eight amino-acid differences within three leucine-rich repeats between Pi2 and Piz-t resistance proteins determine the resistance specificity to Magnaporthe grisea. Mol. Plant-Microbe Interact. 19:1216-1228.

62. Zhou, T., Wang, Y., Chen, J. Q., Araki, H., Jing, Z., Jiang, K., Shen, J., and Tian, D. 2004. Genome-wide identification of NBS genes in japonica rice reveals significant expansion of divergent non-TIR NBS-LRR genes. Mol. Genet. Genomics 271:402-415.

63. Zhu, M. L., Wang, L., and Pan, Q. H. 2004. Identification and characterization of a new blast resistance gene located on rice chromosome 1 through linkage and differential analyses. Phytopathology 94:515-519.

64. Zhuang, J. Y., Ma, W. B., Wu, J. L., Chai, R. Y., Lu, J., Fan, Y. Y., Jin, M. Z., Hei, L., and Zheng, K. L. 2002. Mapping of leaf and neck blast resistance genes with resistance gene analog, RAPD and RFLP in rice. Euphytica 128:363-370. 